\title{
Does the Addition of a Dynamic Pedicle Screw to a Fusion Segment Prevent Adjacent Segment Pathology in the Lumbar Spine?
}

\author{
Aygun Hayati, Yaray Osman, Mutlu Muren \\ Department of Orthopaedics/Spine, Spatial Medicabil Hospital, Bursa, Turkey
}

\section{Study Design: Retrospective clinical cohort study.}

Purpose: To investigate whether the combined use of dynamic pedicle screws and polyaxial pedicle screws was effective on adjacent segment pathology (ASP).

Overview of Literature: Various screw and rod models have been recently developed for preventing adjacent segment disease, and hybrid systems have been described along with posterior instrumentation in the fusion segment. In the literature, although the success of dynamic systems has been demonstrated in non-fusion posterior instrumentation, it remains unclear whether the addition of a screw-based dynamic system to a fusion segment would successfully prevent ASP in the long term.

Methods: The study included 101 patients who underwent surgery for degenerative spine diseases between 2007 and 2014 with lumbar stabilization that used either polyaxial pedicle screws alone or polyaxial pedicle screws plus dynamic stabilization screws (with hinged screw heads). These two patient groups were compared using retrospectively obtained postoperative new clinical findings, Oswestry disability index (ODI) scores, visual analog scale (VAS) scores, and radiological data.

Results: The proportion of patients with ASP who were radiologically assessed was low $(p<0.01)$ in the group that underwent lumbar stabilization along with dynamic screws. Treatment outcomes were clinically successful in both groups according to ODI and VAS scores, and no significant difference was determined between the groups in terms of clinical ASP ( $p>0.05)$.

Conclusions: Although the combined use of dynamic screws and the static system was radiologically found to be effective for preventing ASP in patients who underwent lumbar fusion with posterior instrumentation, it did not completely eliminate ASP or result in a significant improvement in clinical ASP.

Keywords: Adjacent segment pathology; Dynamic fixation; Lumbar spine; SCREW-based dynamic system; Degenerative disk

\section{Introduction}

A possible explanation for adjacent segment pathology (ASP) is that when segments undergo fusion, the adjacent segments have to compensate for the lost range of move- ment, resulting in exposure of those segments to overload and shear forces [1-4]. Various screw and rod models have been recently developed for preventing adjacent segment disease, and hybrid systems have been described along with posterior instrumentation in the fusion segment [5-8].

Received Nov 25, 2016; Revised Feb 6, 2017; Accepted Feb 19, 2017

Corresponding author: Hayati Aygun

Department of Orthopaedics/Spine, Spatial Medicabil Hospital, 16400 Bursa, Turkey

Tel: +90-532-694-5552, Fax: +90-224-243-5533, E-mail: hayatiaygun@gmail.com 
Table 1. Patient characteristics and intraoperative datum

\begin{tabular}{lccc} 
Characteristic & PPS & PP+DPS & $p$-value \\
Age & $54.2 \pm 5.11$ & $52 \pm 6.02$ & $>0.05$ \\
Sex (female/male) & $34 / 25$ & $19 / 23$ & $>0.05$ \\
\hline Smoker & 7 & 4 & $<0.05$ \\
BMl (female/male, $\mathrm{kg} / \mathrm{m}^{2}$ ) & $23.12 \pm 2.3 / 22.45 \pm 3.12$ & $23.43 \pm 3.4 / 21.75 \pm 1.45$ & $>0.05$ \\
Operative time (min) & $178 \pm 57$ & $203 \pm 48$ & $>0.05$ \\
Blood loss $(\mathrm{mL})$ & $624 \pm 245$ & $712 \pm 316$ & $>0.05$ \\
\hline
\end{tabular}

PPS group, poly axial pedicle screw group; PP+DPS group, combine poly axial and dynamic pedicle screw group.

Although the success of dynamic systems has been demonstrated in non-fusion posterior instrumentation, whether hybrid systems are successful in preventing ASP remains debatable $[1,9,10]$. This study investigated whether the addition of dynamic pedicle screws from the screw head to the fusion segment was effective in preventing adjacent segment disease in patients who underwent lumbar segmental spinal fusion. For this evaluation, results were compared between posterolateral fusion (PLF) patients stabilized only with a stable posterior instrumentation system and patients for whom dynamic screws were added to the posterior instrumentation system from the caudal and cranial ends.

\section{Materials and Methods}

This retrospective clinical and radiological evaluation included 101 patients who underwent lumbar segmental spinal fusion between 2007 and 2014 (Table 1). The patients comprised 53 females and 48 males, with a mean age of 53 years (range, 38-78 years). Lumbar fusion involved either adding dynamic pedicle screws to adjacent segments (distal and proximal) or instrumentation only with polyaxial pedicle screws (Table 2). For longsegment instrumentation, the rods were first bent lordotically. Patients were positioned for a favorable lordosis. To achieve strong implantation, long and thick screws (6.0 hydroxyapatite-coated screws) were used and implanted at an angle of approximately $15-20$ degrees relative to the sagittal plane to protect the facet joints. This position can reduce the risk for a lateral or medial perforation, and it avoids the hinge joints of the screws being in parallel to one another. This implantation technique also allows micromobility in the sagittal plane (flexion and extension), thereby avoiding rigid fixation and excessive mobility in the sagittal plane at the tip and bottom of the fused seg-
Table 2. Etiology of the patients

\begin{tabular}{lcc} 
Etiology & PPS & PP+DPS \\
\hline Degenerative scoliosis & 8 & 6 \\
Degenerative spondylolisthesis & 7 & 6 \\
\hline Istmic spondylolisthesis & 3 & 3 \\
\hline Post surgical flatback & 5 & 6 \\
\hline Degenerative disc disease & 10 & 9 \\
Spinal stenosis & 15 & 7 \\
Recurrent disc herniation & 11 & 5 \\
Total & 59 & 42 \\
\hline
\end{tabular}

PPS group, poly axial pedicle screw group; PP+DPS group, combine poly axial and dynamic pedicle screw group.

ment. The screws were deeply implanted in the pedicle to minimize the leverage forces acting on the implants. Patients were excluded from the study if they were suffering from comorbidities (diabetes, severe thoracolumbar osteoporosis, osteomyelitis, severe cardiac and pulmonary diseases, or malignancies) or preoperative ASP, or if they underwent revision surgery. All patients were preoperatively evaluated using the Oswestry disability index (ODI) and visual analog scale (VAS) scoring systems.

The patients were analyzed in two groups poliaxial pedicle screw (PPS) and poliaxial pedicle screw+dynamic pedicle screw (PPS+DPS) according to the technique used. In the PPS group, patients $(n=59)$ underwent PLF after laminectomy and decompression with demineralized bone matrix (DBM) grafts and polyaxial pedicle screw rods plus interbody polyetheretherketone cages and DBM grafts (Johnson \& Johnson, Somerville, NJ, USA). A total of 127 segments were fused (Table 3). In the PPS+DPS group, patients $(n=42)$ also underwent lumbar spinal fusion; in addition, dynamic pedicle screws with hinged screw heads (cosmic, Ulrich GmBH \& Co. KG, Ulm, 
Table 3. Fusion levels and the ASP levels according to groups

\begin{tabular}{|c|c|c|c|}
\hline PPS & Patients & RASP & CASP \\
\hline \multicolumn{4}{|c|}{ Main fused segments } \\
\hline $\mathrm{L} 2-\mathrm{L} 3$ & 3 & 0 & 0 \\
\hline L3-L4 & 7 & 1 & 0 \\
\hline L4-L5 & 11 & 2 & 0 \\
\hline L5-S1 & 12 & 3 & 0 \\
\hline L4-S1 & 15 & 3 & 1 \\
\hline L3-S1 & 4 & 2 & 1 \\
\hline L2-S1 & 4 & 2 & 2 \\
\hline L1-S1 & 2 & 1 & 1 \\
\hline Total & 58 & 14 & 5 \\
\hline \multicolumn{4}{|l|}{$\mathrm{PP}+\mathrm{DPS}$} \\
\hline \multicolumn{4}{|c|}{ Added dynamic segments } \\
\hline $\mathrm{L} 1-\mathrm{L} 2, \mathrm{~L} 3-\mathrm{L} 4$ & 2 & 0 & 0 \\
\hline $\mathrm{L} 2-\mathrm{L} 3, \mathrm{~L} 4-\mathrm{L} 5$ & 3 & 1 & 1 \\
\hline L3-L4, L5-S1 & 6 & 2 & 1 \\
\hline L4-L5 & 9 & 3 & 1 \\
\hline L3-L4 & 9 & 1 & 0 \\
\hline $\mathrm{L} 2-\mathrm{L} 3$ & 7 & 1 & 0 \\
\hline $\mathrm{L} 1-\mathrm{L} 2$ & 4 & 0 & 0 \\
\hline $\mathrm{T} 12-\mathrm{L} 1$ & 2 & 0 & 0 \\
\hline Total & 42 & 8 & 3 \\
\hline
\end{tabular}

ASP, adjacent segment pathologies; PPS group, poly axial pedicle screw group; RASP, radiological adjacent segment pathologie; CASP, clinical adjacent segment pathologies; PP+DPS group, combine poly axial and dynamic pedicle screw group.

Germany) were added to the adjacent segments at the end of the fused segment (Figs. 1, 2). The hinged head joints of the dynamic screws allowed 20 degrees of movement in one direction. The dynamic screws were added to the caudal and cranial ends of the fusion site (Table 2); in case the screws did not reach the sacral region $(n=11)$, polyaxial screws were used after posterior instrumentation. In the remaining cases $(\mathrm{n}=31)$ in which the sacral spine was involved in the fusion, dynamic screws were added only to the cranial end of the fusion site. In this group, 84 segments were fused and 53 segments were added to these segments as dynamic segments (Table 3 ).

Operating time and blood loss were compared between the groups. Radiological and clinical follow-up data were obtained from hospital records for the 6-month postoperative period. Computed tomography was used to determine the fusion rate at 12 and 24 months. Clinical adjacent segment pathologies (CASP) were evaluated ac-

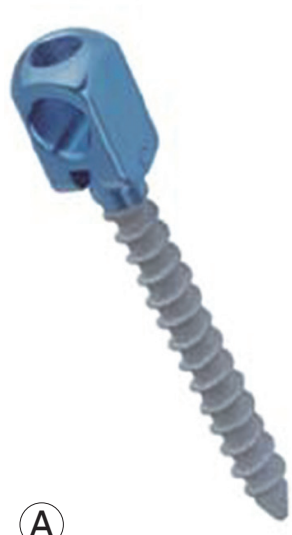

(A)

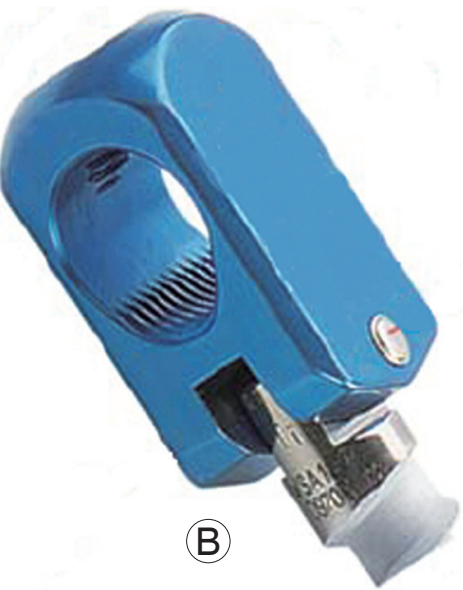

Fig. 1. (A, B) Dynamic pedicle screw (Cosmic; data from Ulrich $\mathrm{GmBH}$ \& Co., with Permitted by Ulrich GmBH \& Co. KG).
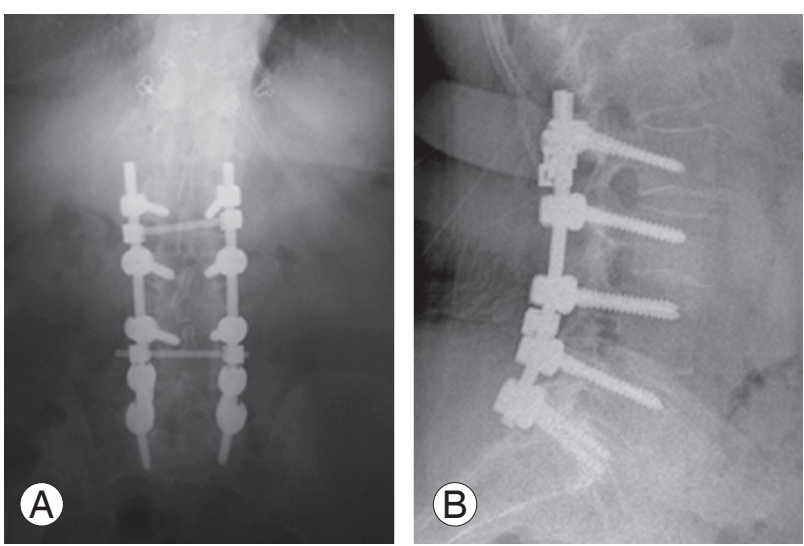

Fig. 2. (A, B) Combination of a pedicle polyaxial screw and a dynamic pedicle screw in the lumbar spine.

cording to whether there was symptomatic spinal stenosis, mechanical low back pain, or sacral or coronal imbalance after the procedures. Radiological adjacent segment pathologies (RASPs) were evaluated according to the Weiner classification. Statistical analysis of the results was performed using the SPSS (SPSS Inc., Chicago, IL, USA) statistics software program. A $p$-value of $<0.05$ was considered to be statistically significant.

This study was approved by the Local Ethics Committee and was performed according to the ethical standards of the 1964 Declaration of Helsinki.

\section{Results}

The patients were followed up for an average of 79 months (range, 55-90 months). In the PPS group, where dynamic screws were not used, radiological assessment found ASP 
in caudal segments in $14 \%(8 / 59)$ of patients and in cranial segments in $10 \%(6 / 59)$ of patients (Table 3$)$. In the PPS+DPS group, where the dynamic screw combination was used, radiological assessment revealed ASP in caudal and cranial segments in $11.9 \%(5 / 42)$ and $7 \%(3 / 42)$ of patients, respectively. This difference was statistically significant: RASP was less frequently observed in patients for whom a dynamic screw combination was used $(p<0.05)$ (Table 3). Evaluation of CASP showed symptomatic findings in $8 \%(5 / 49)$ of patients in the PPS group and in 7\% (3/42) of patients in the PPS+DPS group. There was no significant difference between the two groups in terms of CASP $(p>0.05)$ (Table 3$)$.

Successful clinical outcomes were observed in both the groups, with no significant differences between the groups with respect to ODI and VAS scores ( $p>0.05$ ) (Tables 4-6). There were no significant differences between the groups with regard to pre- and postoperative global and regional alignment data. Equally good results were achieved in both groups in terms of regional alignment (Table 7). No significant differences were found between the groups regarding fusion rates ( $89 \%$ in both the groups), operating times, or blood loss (all $p>0.05$ ). Furthermore, there were no significant differences between the groups in terms of complications (Table 8). Pseudarthrosis occurred in four patients; one underwent surgical revision and the other three were followed up without any clinical complaints. Screw loosening occurred in two patients in the PPS

Table 4. Oswestry disability index and visual analog scale scores of PPS group

\begin{tabular}{lcc} 
Variable & Oswestry disability index & Visual analog scale \\
Preoperative & 47.3 & 8.2 \\
Postoperative & 9.23 & 2.3 \\
$p$-value & $<0.001$ & $<0.001$ \\
\hline
\end{tabular}

PPS, polyaxial pedicle screw group.

Table 5. Oswestry disability index and visual analog scale scores of PPS+DPS group

\begin{tabular}{lcc} 
Variable & Oswestry disability index & Visual analog scale \\
Preoperative & 51.2 & 8.8 \\
Postoperative & 8.16 & 2.4 \\
$p$-value & $<0.001$ & $<0.001$ \\
\hline
\end{tabular}

PPS, polyaxial pedicle screw; DPS, dynamic pedicle screw.

Table 6. Comparison of Oswestry disability index and visual analog scale scores of PPS and PP+DPS groups

\begin{tabular}{lccc} 
Pain indices & Group 1 & Group 2 & $p$-value \\
Oswestry disability index & 9.23 & 8.16 & $>0.05$ \\
Visual analog scale & 2.3 & 2.4 & $>0.05$ \\
\hline
\end{tabular}

PPS, polyaxial pedicle screw; PP+DPS group, combine poly axial and dynamic pedicle screw group.

Table 7. Comparison of lumbar lordozis and sagittal alignment polyaxial pedicle screw and polyaxial pedicle scerew+dynamic pedicle screw groups preoperatively-postoperatively

\begin{tabular}{|c|c|c|c|c|c|}
\hline \multirow{2}{*}{ Parameters } & \multicolumn{2}{|c|}{ Group 1} & \multicolumn{2}{|c|}{ Group 2} & \multirow{2}{*}{$p$-value } \\
\hline & Preoperative & Postoperative & Preoperative & Postoperative & \\
\hline Lumbar lordozis & 19.3 & 32.6 & 18.7 & 32.2 & $>0.05$ \\
\hline Pelvic tilt & 18.31 & 14.40 & 17.78 & 14.16 & $>0.05$ \\
\hline Pelvic incidence & 50.19 & 51.23 & 50.81 & 52.13 & $>0.05$ \\
\hline Sagittal vertical axis & 4.7 & 4.2 & 4.9 & 4.4 & $>0.05$ \\
\hline
\end{tabular}


Table 8. Complications in both groups

$\begin{array}{lcc}\text { Complications } & \text { PPS } & \text { PP+DPS } \\ \text { Dural tear } & 1 & 1 \\ \text { Radiculopathy } & 2 & 1 \\ \text { Infection } & 1 & 0 \\ \text { Loosening } & 2 & 1 \\ \text { Pseudoarthrosis } & 3 & 1\end{array}$

PPS group, poly axial pedicle screw group; PP+DPS group, combine poly axial and dynamic pedicle screw group.

group and in one patient in the PPS+DPS group. No additional surgical intervention was administered to patients who developed ASP.

\section{Discussion}

ASP is a significant long-term complication that affects the success of posterior instrumentation and fusion [1-4]. Many non-fusion stabilization systems using screws and rods have been developed to prevent this complication $[8,9]$. These systems have been mainly classified according to the screw, rod, and interspinous implants used, with reports that these provide many individual advantages $[10,11]$.

ASP occurs because of the transmission of compensatory compression, flexion-extension, and shear forces from the fusion segment to the disc and facet joints of adjacent segments, resulting in excessive loading in those areas. The effect of this excessive loading must be reduced or eliminated, and there has been much discussion in the literature regarding systems that address this. It has been reported that the use of interspinous devices leads to different problems through reducing lordosis in the lumbar region and increasing loading on the spinous processes [6-10]. Dynamic stabilization devices have been approved for use as adjuncts to spinal fusion. When used in this manner, these devices add stability to the spine and help maintain a proper spinal alignment during fusion. The advantage of this over traditional fusion is that the dynamic stabilization construct is much less rigid, possibly allowing small amounts of movement and helping prevent the rapid degeneration of adjacent disks and facets. This technique is sometimes referred to as "soft fusion."

In this study, we used a cosmic pedicle screw rod system, wherein the screw head is connected to the threaded section via a hinged joint. Similar to the hinge on a door, the hinged joint permits motion in only one plane. When screws are connected using longitudinal rods, there is a high degree of stability in relation to the rotational forces, as well as micromobility in the sagittal plane. Thus, the system reduces compressive loadings on the adjacent segment through the dynamic screw head but allows flexion and extension to a certain extent [12-14], although the segment remains stable during rotation movement and shear forces. This condition may be increased to rotational movement in the adjacent segment to which the dynamic screw was added. Flexible rods may be more advantageous because they allow rotational movement in addition to flexion and extension, preserving the natural movements of the adjacent segment. Thus, the use of flexible rods to reduce loading on the adjacent segment appears to be a reasonable procedure [15-18].

However, global and regional spinal malalignment play an important role in ASP. Besides decompression and stabilization, an additional aim of surgery is to reestablish the anatomical spinopelvic parameters. Motion segment fusion is not the only cause of ASD; fusion plus the presence of malalignment end fusion segment appears to be a major factor in end fusion stresses that result in ASP. However, in our study, the spinal alignments and corrections were within the normal range, and there were no differences between the two groups in pre- and postoperative global and regional spinal alignment parameters (Table 7).

In this study, dynamic screws placed in the segments adjacent to the rigid stabilization segment played a limited role in preventing RASP. However, no such finding was obtained for clinical data. Importantly, a dynamic pedicle screw with a hinged screw head could not completely eliminate ASP, as shown by RASP being observed in eight (14\%) of the patients in the PPS+DPS group (Table 3). This may have occurred because of screw heads providing dynamization, enabling partial dynamic stabilization by not allowing rotational movements in the adjacent segment. Hinged pedicle screws behave like polyaxial pedicle screws when subjected to rotational and lateral bending movements or loading. In addition, clinical data showed no significant difference obtained between the two groups in terms of CASP, and no surgical interventions were required for he patients who developed CASP.

Clinical and radiological ASPs are two different entities $[1,9]$. Clinical symptoms may not be observed in low grades of RASP (Grades 1 and 2) and even in some cases 
of Grade 3 RASP. There is still no suitable system for the clinical evaluation of ASP. Therefore, in this study, CASP formation was evaluated only as postoperative degenerative changes in adjacent segments and related symptoms.

\section{Conclusions}

The study results have shown that in patients undergoing instrumented fusion to address degenerative spine diseases, the addition of a dynamic pedicle screw to the lower and upper ends of the posterior instrumentation segments appears to be effective in terms of RASP. However, with respect to clinical findings (CASP), there was no significant difference between the cases where only posterior instrumentation was used and those where a polyaxial and dynamic screw combination was applied $(p>0.05)$ (Table 3). Although the results were not statistically significant, the addition of a dynamic screw had beneficial effects on the operation process and amount of bleeding. However, the addition of a dynamic screw to the posterior instrumentation system increased the length of the instrument and the number of segments that were affected.

\section{Conflict of Interest}

No potential conflict of interest relevant to this article was reported.

\section{References}

1. Norvell DC, Dettori JR, Skelly AC, Riew KD, Chapman JR, Anderson PA. Methodology for the systematic reviews on an adjacent segment pathology. Spine (Phila Pa 1976) 2012;37(22 Suppl):S10-7.

2. Kaito T, Hosono N, Mukai Y, Makino T, Fuji T, Yonenobu K. Induction of early degeneration of the adjacent segment after posterior lumbar interbody fusion by excessive distraction of lumbar disc space. J Neurosurg Spine 2010;12:671-9.

3. Lee MJ, Dettori JR, Standaert CJ, Ely CG, Chapman JR. Indication for spinal fusion and the risk of adjacent segment pathology: does reason for fusion affect risk? A systematic review. Spine (Phila Pa 1976) 2012;37(22 Suppl):S40-51.

4. Lund T, Oxland TR. Adjacent level disk disease: is it really a fusion disease? Orthop Clin North Am 2011;42:529-41.
5. Kumar A, Beastall J, Hughes J, et al. Disc changes in the bridged and adjacent segments after Dynesys dynamic stabilization system after two years. Spine (Phila Pa 1976) 2008;33:2909-14.

6. Sengupta DK. Dynamic stabilization devices in the treatment of low back pain. Neurol India 2005;53:466-74.

7. Liu CL, Zhong ZC, Shih SL, Hung C, Lee YE, Chen CS. Influence of Dynesys system screw profile on adjacent segment and screw. J Spinal Disord Tech 2010;23:410-7.

8. Maserati MB, Tormenti MJ, Panczykowski DM, Bonfield CM, Gerszten PC. The use of a hybrid dynamic stabilization and fusion system in the lumbar spine: preliminary experience. Neurosurg Focus 2010;28:E2.

9. Cheh G, Bridwell KH, Lenke LG, et al. Adjacent segment disease followinglumbar/thoracolumbar fusion with pedicle screw instrumentation: a minimum 5-year follow-up. Spine (Phila Pa 1976) 2007;32:22537.

10. Kim CH, Chung CK, Jahng TA. Comparisons of outcomes after single or multilevel dynamic stabilization: effects on adjacent segment. J Spinal Disord Tech 2011;24:60-7.

11. Kanayama M, Togawa D, Hashimoto T, Shigenobu $\mathrm{K}$, Oha F. Motion-preserving surgery can prevent early breakdown of adjacent segments: comparison of posterior dynamic stabilization with spinal fusion. J Spinal Disord Tech 2009;22:463-7.

12. Kaner T, Sasani M, Oktenoglu T, Ozer AF. Dynamic stabilization of the spine: a new classification system. Turk Neurosurg 2010;20:205-15.

13. von Strempel A. Dynamic posterior stabilization with the cosmic system. Oper Orthop Traumatol 2010;22:561-72.

14. Stoffel M, Behr M, Reinke A, Stuer C, Ringel F, Meyer B. Pedicle screw-based dynamic stabilization of the thoracolumbar spine with the Cosmic-system: a prospective observation. Acta Neurochir (Wien) 2010;152:835-43.

15. Coe JD, Kitchel SH, Meisel HJ, Wingo CH, Lee SE, Jahng TA. NFlex dynamic stabilization system: twoyear clinical outcomes of multi-center study. J Korean Neurosurg Soc 2012;51:343-9.

16. Wang JC, Arnold PM, Hermsmeyer JT, Norvell DC. Do lumbar motion preserving devices reduce the risk of adjacent segment pathology compared with fusion 
surgery? A systematic review. Spine (Phila Pa 1976) 2012;37(22 Suppl):S133-43.

17. Lee SE, Park SB, Jahng TA, Chung CK, Kim HJ. Clinical experience of the dynamic stabilization system for the degenerative spine disease. J Korean Neurosurg Soc 2008;43:221-6.
18. Hoppe S, Schwarzenbach O, Aghayev E, Bonel H, Berlemann U. Long-term outcome after monosegmental L4/5 stabilization for degenerative spondylolisthesis with the dynesys device. Clin Spine Surg 2016;29:72-7. 